\title{
SENSIBILIDAD A LA ANSIEDAD VERSUS RASGO DE ANSIE- DAD EN LA PREDICCIÓN DEL NIVEL DE MIEDOS
}

\author{
Bonlfacio Sandin \\ Rosa M. Valiento \\ Paloma Chorot \\ M.Ángel Santed \\ Lourdes Lostao*
}

Facultad de Psicologla. Universidad Nacional de Educación a Distancia. UNED. Madrid Universidad Püblica de Navarra. Pamplona*

\section{RESUMEN}

Este estudio analiza tres hipotesis interrelacionadas sobre la separación entre la sensibilidad a la ansiedad (SA) y el rasgo de ansiedad y sobre la capacidad que poseen las dimensiones especificas de SA para predecir el miedo. Se aplicó la versión española de la escala de sensibilidad a la ansiedad ASI (Anxiety Sensitivity Index; Reiss et al., 1986; Sandin et al., 2004) junto a otras pruebas de ansiedad, depresión y personalidad a una muestra de 910 estudiantes universitarios. El análisis de la ASI se efectuó tanto a nivel de la escala total como a nivel de las tres subescalas (somática, cognitiva y social). Los resultados indicaron que la ASI resulto ser el mejor predictor de los miedos; las restantes variables resultaban insignificantes cuando se controlaba el efecto de la ASI. La subescala ASI-somática predecía más especificamente los miedos agorafóbicos y los del tipo sangre-inyección-daño, mientras que la subescala ASI- 
social lo era para los miedos sociales. Los datos son discutidos en relación con (1) la separación entre la SA y el rasgo de ansiedad, (2) la predicción diferencial de la $S A, y$ (3) la validez predictiva de la versión española de la ASI.

Palabras clave: SENSIBILDAD A LA ANSIEDAD, ASI, MIEDOS, RASGO DE ANSIEDAD, AFECTIMDAD, PERSONALIDAD.

\section{SUMMARY}

This study evaluated three interrelated hypotheses conceming separation between anxiety sensitivity (AS) and trait-anxiety and how specific AS dimensions predicts fear. A sample of 910 undergraduates completed a psychometric assessment package that included the Spanish version of the Anxiety Sensitivity Index (ASl; Reiss et al., 1986; Sandin et al., 2004) and measures of fears, anxiety, depression and personality. AS was analyzed at the levels of the total scale and the three ASI-subscales (Physical, Cognitive-, and Social-Concems). The ASI was found to be statistically the best predictor, accounting for fears above and beyond the variance accounted for by the remaining variables. The Physical Concems subscale was predictive of agoraphobic and blood-injection-injury fears, whereas the Social Concems subscale predicted social fears. Findings were discussed in relation to (1) separation between AS and trait-anxiety, (2) differential predictive validity of AS, and (3) predictive validity of the Spanish version of the ASI.

Key words: ANXIETY SENSITIVITY, ASI, FEARS, TRAIT ANXIETY, AFFECTIVITY, PERSONALITY.

\section{INTRODUCCIÓN}

La sensibilidad a la ansiedad (SA) ha sido definida como el temor a los sintomas de ansiedad. La SA, aunque parece constituir un marcador importante del trastomo de pánico (Chorot, Sandin, Valiente, Santed y 
Romero, 1997; McNally, 1996; Taylor, 1999), también se ha vinculado de forma especifica con otros trastornos y manifestaciones de la ansiedad, incluidos los miedos y las fobias (Reiss, 1991; Sandín, 1997, 1999; Taylor, 1999). Los miedos son fenómenos muy comunes, no sólo entre la población clínica, sino también en la población general, especialmente en la población infantojuvenil (Pelechano, 1981, 1984; Sandín, Chorot, Valiente y Santed, 1998; Sandin, Chorot, Valiente, Santed y Sánchez-Arribas, 1999a, 1999b; Valiente, Sandín, Chorot y Tabar, 2002, 2003). Se ha sugerido que, tanto en población adulta (Lilienfeld, 1997; Reiss, Peterson, Gursky y McNally, 1986; Sandin, 2005) como en población infantojuvenil (Sandlin, Chorot, Valiente y Santed, 2002b), la SA puede proporcionar un nivel significativo de predicción del nivel de miedos adicional a la predicción proporcionada por el rasgo de ansiedad $u$ otras variables de personalidad (p.ej., la emocionalidad negativa).

La SA es diferente al rasgo de ansiedad. Mientras que el rasgo de ansiedad es la tendencia a experimentar ansiedad ante cualquier situación estresante, la SA es la tendencia a experimentar miedo (o ansiedad) a los propios sintomas de ansiedad. Muchas personas tienen un elevado rasgo de ansiedad, pero no experimentan miedo a las sensaciones de ansiedad. Aunque a veces se ha cuestionado tal separación conceptual (Lilienfeld, 1996), la SA no debería confundirse con el rasgo de ansiedad. Los datos basados en análisis factorial de Taylor et al. (1991) y Sandin, Chorot y McNally (2001) han demostrado empiricamente la separación entre ambos constructos. Desde el punto de vista predictivo, la evidencia de que la SA pueda predecir el miedo y el pánico de forma más precisa e independiente a la predicción que aporta el rasgo de ansiedad (Sandín et al., 2002b) constituirla, asi mismo, una prueba a favor de la distinción entre la SA y el rasgo de ansiedad. En esta línea, nuestro grupo ha proporcionado datos con población de niños y adolescentes que sugieren una relación más estrecha de los miedos con la SA que con el rasgo de ansiedad (Sandín et al., 2002b; Valiente, Sandin y Chorot, 2002a, 2002b).

Aunque inicialmente se habia hipotetizado que la SA era un constructo unidimensional, recientemente se han acumulado datos a favor de una estructura jerárquica, constituida por tres factores de primer orden y un factor de orden superior (Stewart, Taylor y Baker, 1997; Zinbarg, Barlow y Brown, 1997; Sandín, Chorot, Santed y Valiente, 
2002a; Sandín, Chorot, Valiente, Santed y Lostao, 2004). La existencia de tres componentes de SA, por otra parte, es congruente con el supuesto básico de que la SA se refiere al miedo a las sensaciones fisicas de ansiedad, derivado de la creencla de que dichas sensaciones poseen posibles consecuencias peligrosas de tipo somático, psicológico o social (Reiss et al., 1986).

Zinbarg et al. (1997) y Stewart et al. (1997) publicaron los primeros trabajos, el primero con sujetos clínicos y el segundo con estudiantes universitarios, que sugerian una estructura de ASI consistente en tres factores primarios (denominados por Zinbarg et al. como Physical Concerns, Mental (ncapacitation Concerns y Social Concerns), los cuales saturaban en un factor general de SA (estructura jerárquica). Recientemente nuestro grupo ha validado este modelo jerárquico mediante análisis factorial confirmatorio con una muestra amplia de participantes no clínicos (Sandin et al., 2004). Este trabajo, que constituye el primer estudio sobre la estructura jerárquica de la SA con población no clínica basado en análisis de ecuaciones estructurales, ha supuesto una extensión de los datos confirmatorios iniciales obtenidos por Zinbarg et al. (1997) a partir de una muestra clínica, y sugiere que el modelo jerárquico de la SA también es válido para la población no clínica.

La existencia de un modelo jerárquico de la SA posee importantes implicaciones para la psicopatología y el tratamiento de la ansiedad (Sandín et al., 2004). En primer lugar, el hecho de que existan tres componentes de SA que son al menos parcialmente distintos (SAsomática, SA-cognitiva, y SA-social), permite describir este constructo psicopatológico de forma diferente a como se ha venido haciendo (i.e., unidimensional). Permite también derivar subescalas de SA y relacionar estas dimensiones primarias con otros constructos psicólogos de la ansiedad y otras características psicopatológicas. Mientras que ciertos componentes de SA, o el factor general, pueden estar etiológicamente más implicados en unos trastomos de ansiedad que en otros, también pueden ser de gran utilidad para estudios sobre la predicción y evolución de los trastornos de ansiedad. Por ejemplo, mientras que la SA-somática podría estar más relacionada con el trastomo de pánico (Zinbarg et al., 1997; Brown, Smits, Powers y Telch, 2003), la SA-social parece presentar una relación más estrecha con la fobia social (Zinbarg et al., 1997). También se ha sugerido que la 
SA-cognitiva presenta una cierta asociación con la depresión mayor (Taylor, Koch, Woody y McLean, 1996).

A pesar de los múltiples estudios que se han llevado a cabo con la ASI, la información sobre validez predictiva generalmente se ha basado en la conceptuación unidimensional de la ASI, esto es, en las puntuaciones globales obtenidas a partir de esta escala. No obstante, la adopción del reciente modelo jerárquico justifica la necesidad de llevar a cabo nuevos estudios sobre las propiedades y caracterlsticas de la ASI teniendo en cuenta tanto sus diferentes dimensiones como la puntuación total en SA. En nuestro reciente estudio (Sandín, Valiente, Chorot y Santed, 2005), hemos proporcionado datos a favor de la fiabilidad y validez de la ASI teniendo en cuenta las tres dimensiones sugeridas [véase este estudio para obtener información sobre datos normativos, consistencia interna y validez (convergente/divergente) de la ASI (para la escala total y para las tres subescalas)].

Medlante el presente estudio, que constituye una continuación del referido arriba (Sandin et al., 2005), pretendemos analizar la diferenciación entre la SA y el rasgo de ansiedad, asI como también examinar la validez predictiva de la ASI. En nuestra primera hipótesis postulamos que la contribución única (en los niveles de miedos) de la SA debería ser superior a la del rasgo de ansiedad. De acuerdo con nuestra segunda hipótesis, la ASI debería predecir el nivel de miedos mejor que el rasgo de ansiedad u otras variables emocionales o de personalidad (i.e., ansiedad, depresión, afecto negativo, neuroticismo, afecto positivo, extraversión y psicoticismo). Finalmente (tercera hipotesis), las dimensiones de la ASI (somática, cognitiva, y social) deberian predecir diferencialmente los tipos de miedos (agorafóbicos, sociales, y sangre-inyección-daño) (i.e., la SAsocial deberla predecir más especificamente los miedos sociales, mientras que la SA-somática deberla predecir mejor los miedos agorafóbicos y sangre-inyección-daño).

\section{MÉTODO}

\section{Partlclpantes}

La muestra total fue de 910 estudiantes universitarios pertenecientes a universidades de Madrid y Pamplona. La muestra estaba constituida 
por 618 mujeres $(68 \%)$ y 292 varones ( $32 \%)$. No habla diferencias entre ambos grupos en edad; la media de edad de las mujeres era de 20,6 $(D T=2,3)$ y la del grupo de hombres de $20,7(D T=2,4)$. El rango de edad para la muestra total fue de 18 a 36 años.

\section{Pruebas psicológicas}

Los participantes cumplimentaron de forma colectiva (en grupos de 20-50 alumnos) en diversos centros docentes universitarios de Madrid y Pamplona los siguientes cuestionarios de autoinforme:

Indice de Sensibilldad a la Ansiedad (Anxiety Sensitivity Index, ASI] (Peterson y Reiss, 1992). Se aplicó la versión española de Sandin y Chorot (Sandin, Valiente y Chorot, 1999; Sandin et al., 2004). Al igual que la versión original, consta de 16 items y mantiene su misma estructura general y el mismo formato de respuesta. El participante debe indicar el grado en que experimenta cada uno de los 16 enunciados según una escala tipo Likert, pudiendo variar entre "Nada en absoluto" (0) y "Muchisimo» (4). Los items hacen referencia a reacciones de miedo/ansiedad ante la experiencia de sintomas de tipo somático (p.ej., «Me asusto cuando mi corazón late de forma rápida»), sintomas observables socialmente («Para mi es importante no dar la impresión de estar nervioso") ), y sintomas de descontrol cognitivo (p.ej., «Me asusto cuando soy incapaz de mantener mi mente concentrada en una tarea»). En anteriores publicaciones sobre la ASI se ha puesto de relieve que esta prueba posee excelentes propiedades psicométricas, tanto con población clínica como no clínica (Sandín et al., 1996, 2001, 2004). Basándonos en las dimensiones de la ASI descritas por Sandín et al. (2004), aparte de la obtención de una puntuación general, a partir de la ASI derivamos las tres subescalas siguientes: (1) ASI-somática (Items $4,6,8,9,10,11$ y 14), (2) ASI-cognitiva (Items 2, 3, 12, 15 y 16), (3) ASI-social (Items 1, 5, 7 y 13).

Cuestionario de Ansiedad Estado-Rasgo STAl [State-Trait Anxiety Inventory]. Se aplicó la versión española adaptada por TEA (Spielberger, Gorsuch y Lushene, 1982). Aplicamos la Parte 2 del STAI, la cual evalúa especificamente el rasgo de ansiedad. Esta parte consta de 20 items, los cuales fueron contestados por los participantes 
indicando cómo se sentlan habitualmente según una escala de 4 niveles que variaba entre 1 ("Casi nunca») y 4 ("Casi siempre»).

Escalas de Afecto Positivo y Negativo PANAS [Positive and Negative Affect Schedule] (Watson, Clark y Tellegen, 1988). Se aplico la versión española, la cual ha sido validada por Sandin, Chorot, Lostao, Joiner, Santed y Valiente (1999). Consta de 20 items, 10 referidos al afecto positivo y 10 al afecto negativo. Cada ítem se puntúa según una escala de 1 («Nada o casi nada») a 5 ("Muchisimo»).

Cuestionario EPQRA [Eysenck Personality Questionnaire RevisedAbbreviated] (Francis, Brown, y Philipchalk, 1992). Se aplico la versión espańola del cuestionario (Sandín, Valiente, Chorot, Olmedo y Santed, 2002b), la cual, como la versión original, consta de 24 items y cuatro subescalas [Extraversión (E), Neuroticismo (N), Psicoticismo (P) y Sinceridad -esta última no ha sido considerada para este estudio)], correspondiendo 6 items a cada una de las subescalas. El formato de respuesta es de SI (1) vs. No (0). Datos sobre la validación de este cuestionario han sido publicados recientemente (Sandin et al., 2002b; Sandín,Valiente, Olmedo, Chorot y Santed, 2002c).

Cuestionario de Miedo [Fear Questionnaire, FQ] (Marks y Mathews, 1979). Se aplicó la versión española de Chorot y Sandín (Sandin, Valiente y Chorot, 1999). El FQ fue desarrollado por Marks y Mathews para obtener una medida abreviada del nivel de miedo, pudiéndose obtener medidas sobre tres tipos de miedos (agorafobia, sociales y sangre-inyección-daño-i.e., del tipo sangre-inyección-daño). Es cuestionario se contesta según una escala de 1 a 8 puntos de evitación. En la presente versión, el FQ consta de 15 items que permite obtener una puntuación total de miedo, asl como también puntuaciones para tres subescalas (FQ-agorafobia, FQ-sociales, y FQ-sangreinyección-danio) (cada subescala consta de 5 items). Los coeficientes de consistencia interna (alfa) de este cuestionario, calculados en el presente estudio, fueron: 0.78 (FQ total), 0,75 (sangre-inyección-daño), 0,64 (sociales) y 0,55 (agorafobia).

Cuestionario de Depresión CESDS [Center for Epidemiological Studies Depresión Scale] (Ensel, 1986). Se aplicó la versión española de Chorot y Sandín (1991). La escala fue diseñada para evaluar los principales componentes de la sintomatologla depresiva (ánimo depresivo, sentimientos de culpa e inutilidad, indefensión y desesperanza, 
pérdida de apetito, perturbación del sueño, y retardo psicomotor). Consta de 20 sintomas que se contestan según una escala de frecuencia [entre 1 («Nunca o pocas veces») y 5 («Casi todo el tiempo»). La CESDS es la primera escala sobre depresión que ha sido validada utilizando conjuntamente muestras comunitarias y clínicas (Ensel, 1986). El coeficiente alfa de Cronbach, calculado en el presente estudio, ha sido de 0,88 .

\section{RESULTADOS}

\section{Medias y desvlaciones típicas}

En la Tabla 1 se indican las medias y desviaciones tipicas correspondientes a las diferentes variables incluidas en el estudio (excepto para las variables de $\mathrm{SA}$; las puntuaciones correspondientes a estas variables han sido publicadas en Sandin et al., 2005). Como puede observarse en dicha tabla, existen diferencias significativas entre los grupos de varones y mujeres para la mayoria de las variables (i.e., para todas las variables excepto extraversion, afecto positivo, miedos agorafóbicos y miedos del tipo sangre-inyección-daño). En todas las variables con diferencias significativas el grupo de mujeres puntúa más elevado que el grupo de varones, excepto en la variable psicoticismo cuyos valores son mayores en el grupo de varones.

Contribución única de la sensibilidad a la ansiedad y del rasgo de ansiedad en los niveles de miedos

Para examinar la contribución única de la SA y del rasgo de ansiedad en los niveles de miedos se calcularon separadamente las correlaciones parciales entre la SA (ASI; puntuación total y puntuaciones en las 3 subescalas) y los niveles de miedos (FQ; puntuación total y puntuaciones en las 3 subescalas) controlando el efecto del rasgo de ansiedad, y entre el rasgo de ansiedad (STAl) y los niveles de miedos controlándose el efecto de la sensibilidad a la ansiedad. Los resultados obtenidos se indican en la Tabla 2.

En términos generales puede observarse que las correlaciones entre el rasgo de ansiedad y el nivel de miedos son muy bajas o insignificantes cuando se controla el efecto de la sensibilidad a la ansiedad. Vemos, por ejemplo, que la correlación entre el STAl y el 
Tabla 1. Medias y desviaciones típicas (entre paréntesis) obtenidas para las diferentes variables en función de los grupos de mujeres $(N=618)$ y de varones $(N=292)$

\begin{tabular}{llll}
\hline & Mujeres & Varones \\
\cline { 2 - 4 } Variables & Media $(D T)$ & Media $(D T)$ & t(908) \\
\hline Variables de personalidad (EPQRA): & & & \\
Neuroticismo (0-6) & $3,3(1,8)$ & $2,8(2,1)$ & $3,95 * \cdots *$ \\
Extraversión (0-6) & $3,7(2,3)$ & $3,4(2,2)$ & 1,81 \\
Psicoticismo (0-6) & $1,6(1,3)$ & $2,1(1,4)$ & $-5,38^{* * *}$ \\
Afecto (PANAS): & & & \\
Afecto negativo (PANAS-N) (10-50) & $22,7(6,8)$ & $20,6(6,5)$ & $3,96 * \cdots *$ \\
Afecto positivo (PANAS-P)(10-50) & $30,4(6,0)$ & $30,3(6,2)$ & 0,23 \\
Rasgo de ansiedad (STAD) (20-80) & $41,1(10,2)$ & $35,7(9,2)$ & $8,04^{* * *}$ \\
Miedos (FQ): & & & \\
FQ-total (15-120) & $49,0(16,1)$ & $45,8(16,6)$ & $2,15 *$ \\
FQ-Agorafobia (5-40) & $10,7(5,4)$ & $11,2(5,2)$ & $-1,11$ \\
FQ-social (5-40) & $18,6(6,7)$ & $16,1(6,8)$ & $4,35^{* * *}$ \\
FQ-sangre-inyección-daf̃o (5-40) & $19,6(9,0)$ & $18,6(9,1)$ & 1,27 \\
Depresión (CESDE) (20-100) & $43,5(11,4)$ & $40,9(10,6)$ & $2,96 * *$ \\
\hline
\end{tabular}

Nota. EPQRA = Eysenck Personality Questionnaire Revised -Abreviado; PANAS = Positive and Negative Affect Schedule; STAI = State Trait Anxiety Inventory; FQ = Fear Questionnaire; CESDS = Center for Epidemiological Studies Depression Scale. ^Entre paréntesis se indican los rangos de puntuaciones para cada variable. - Los grados de libertad pueden cambiar debido a variaciones en el número de sujetos válidos para los cálculos de cada variable.

${ }^{*} p<0,05 ;{ }^{* *} p<0,01 ;{ }^{* * *} p<0,001$ (bilateral).

FQ-total se reduce de 0,26 a 0,10 . Controlándose el efecto de la sensibilidad a la ansiedad, el STAl sólo correlaciona con la dimensión de miedos sociales (la correlación con el FQ-total, aunque es significativa, es prácticamente insignificante). En contraste vemos que, aunque se controle el efecto del rasgo de ansiedad, las distintas variables de sensibilidad a la ansiedad correlacionan de forma moderada con las variables de miedos.

\section{Sensibilidad a la ansiedad y predlcclón del nivel de miedos}

La validez predictiva de la ASI se estudió a partir de la aplicación de varios análisis de regresión múltiple. Para ello se establecieron dos diseños generales de regresión. En un primer diseño, el nivel general 


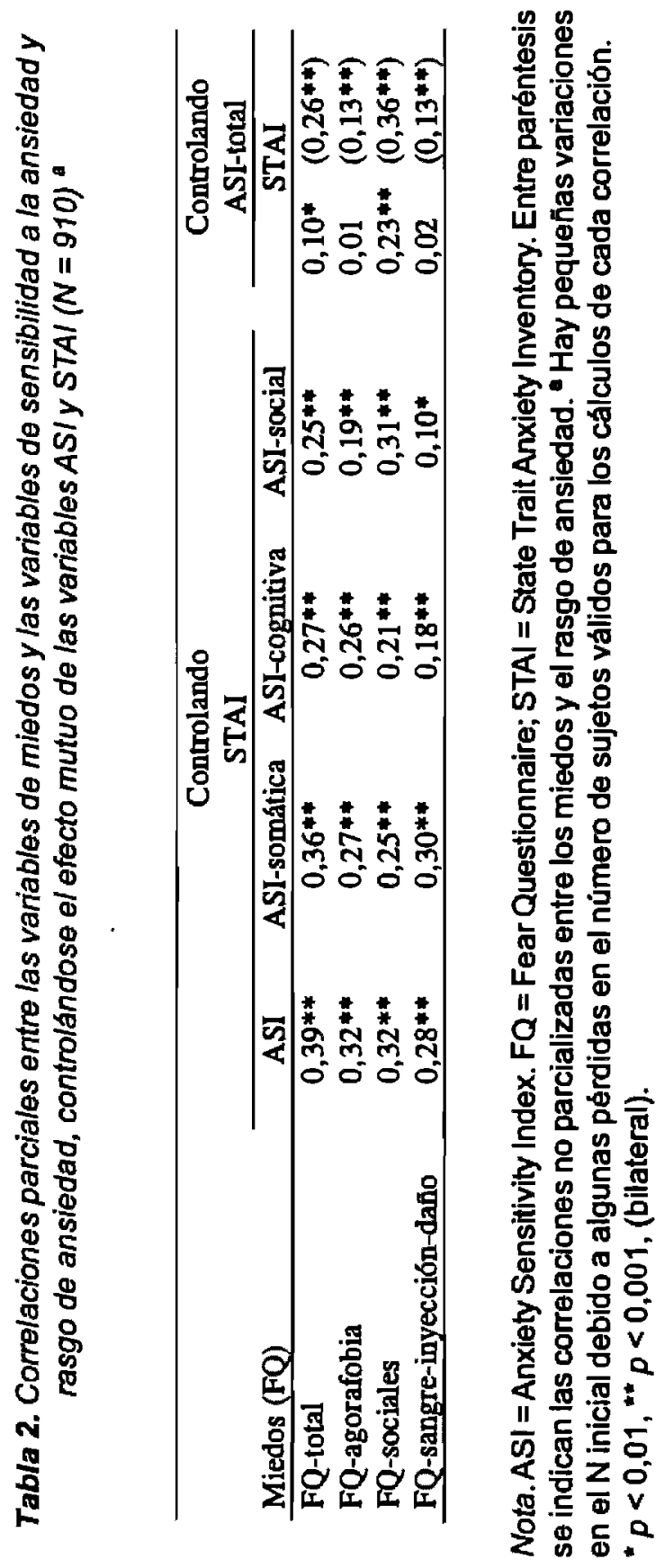


de miedos (FQ-total) fue la variable dependiente y las restantes variables [STAI, PANAS (N y P), EPQRA(N, E y P), y CESDS] actuaron como variables independientes. En un segundo diseño, los predictores fueron las tres subescalas de la ASI (somática, cognitiva y social) y los criterios fueron, sucesivamente, los tres tipos de miedos (agorafobia, sociales, y sangre-inyección-daño). En ambos tipos de diseños se aplicó el método de pasos sucesivos (criterio de entrada: probabilidad para $F £ 0,05$ ). Se comprobó la posible existencia de colinealidad calculándose para ello el valor del VIF (variance inflation factor; Stevens, 1996), no existiendo colinealidad en ninguno de los análisis efectuados, por lo que no se aplicó ningún tipo de control adicional. Los resultados de los análisis de regresión se indican en la Tablas 6 y 7 .

Tabla 3. Análisis de regresión múltiple (método de pasos sucesivos).

Predicción del nivel de miedos (FQ-total) a partir de las variables independientes (predictores) ASI y EPQRA $(E \text { y } N)^{a}(N=910)^{b}$

\begin{tabular}{lllllllr}
\hline $\begin{array}{l}\text { Paso } \\
\text { (modelo) }\end{array}$ & $\begin{array}{l}\text { Predictores } \\
\text { en la ecuación }\end{array}$ & Beta $^{\mathrm{c}}$ & $\mathrm{R}^{2}$ & $\begin{array}{l}\text { Cambio } \\
\mathrm{en}^{2}\end{array}$ & $\begin{array}{l}\mathrm{F} \\
\text { modelo }\end{array}$ & $\begin{array}{l}\mathrm{t} \\
\text { predictores }\end{array}$ & $\mathrm{gl}$ \\
\hline 1 & ASI & 0,43 & 0,18 & 0,18 & $156,9^{* * *}$ & $12,5^{* * *}$ & 1,724 \\
& & & & & & & \\
2 & Efectos generales & & 0,19 & 0,01 & $83,8^{* * *}$ & & 2,723 \\
& ASI & 0,42 & & & & $12,5^{* * *}$ & 723 \\
& EPQRA-E & $-0,10$ & & & & $-3,1^{* *}$ & 723 \\
& & & & & & & \\
3 & Efectos generales & & 0,20 & 0,01 & $58,6^{* * *}$ & & 3,722 \\
& ASI & 0,39 & & & & $11,0^{* * *}$ & 722 \\
& EPQRA-E & $-0,11$ & & & & $-3,2^{* *}$ & 722 \\
& EPQRA-N & 0,10 & & & & $2,6^{*}$ & 722 \\
\hline
\end{tabular}

Nota. ASI $=$ Anxlety Sensitivity Index. FQ = Fear Questionnaire; EPQRA $=$ Eysenck Personallty Questionnalre Revised -Abreviado (E = extraversion, N = neuroticlsmo). - Las restantes varlables Independientes (rasgo de ansiedad, depresión, afecto y psicoticismo) no fueron Incluldas on las ecuaciones de regresión por no satlsfacer el criterlo de entrada. 'Se produjeron algunas varlaciones del $\mathbf{N}$ Inlclal debldo a pérdldas en el número de sujetos válidos para los cálculos estadisticos. " Coeficlente estandarizado.

${ }^{*} p<0,05 ;{ }^{* *} p<0,01$; ** ${ }^{*} p<0,001$. 


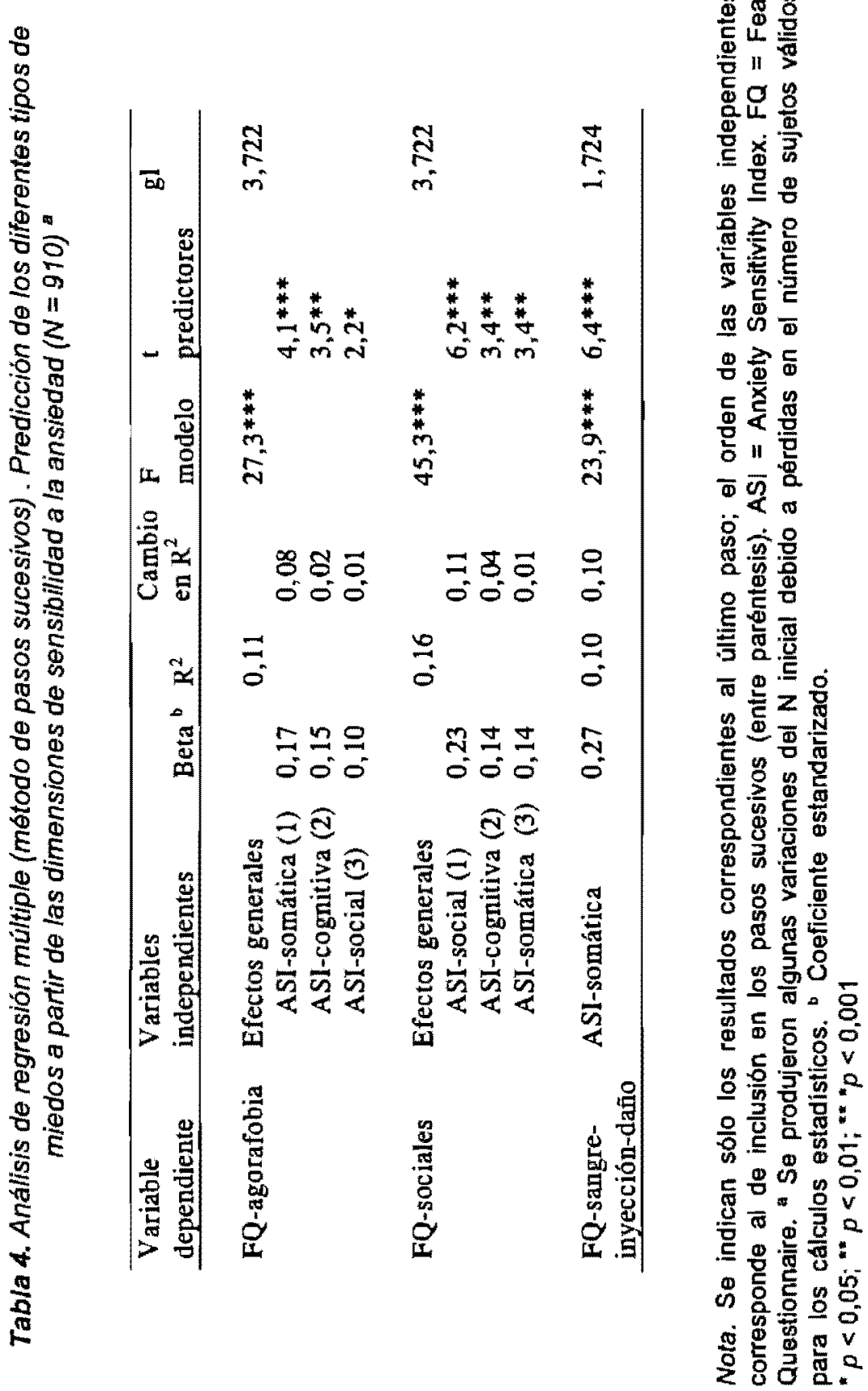


Tras la aplicación del primer diseño, se constató que la ASI predecia de forma significativa el nivel global de miedos (explicaba el $18 \%$ de la varianza total de esta variable), contribuyendo también, aunque de forma limitada, únicamente dos variables (extraversión y neuroticismo) en la predicción del nivel de miedos (no obstante, conjuntamente ambas variables sólo lograban incrementar la varianza en un $2 \%$ ). Merece la pena resaltar que las restantes variables, incluido el rasgo de ansiedad, no proporcionaban ninguna contribución adicional en la explicación de la varianza relativa al nivel de miedos. Es decir, parece claro que, de las 8 variables independientes consideradas conjuntamente en el análisis de regresión, únicamente la ASI predecía de forma relevante el nivel general de miedos (véase la Tabla 3 ).

En la Tabla 4 se indican los resultados obtenidos a partir de los tres análisis de regresión correspondientes respectivamente a los tres criterios (uno para cada subescala de miedos -agorafobia, sociales y sangre-inyección-daño). Mediante estos análisis se pretendía averiguar si las tres dimensiones de SA predeclan diferencialmente las tres dimensiones de miedos. Como puede apreciarse en dicha tabla, los miedos agorafóbicos y sociales son predichos por las tres dimensiones de $S A$, y únicamente los miedos de tipo médico son predichos por una sola dimensión de SA, esto es, la ASI-somática. No obstante, los datos presentados en dicha tabla sugieren también que los miedos sociales son predichos fundamentalmente por la ASI-social, y los miedos agorafóbicos por la ASI-somática. En conclusión, a partir de estos datos podría afirmarse cierto grado de especificidad entre las dimensiones de la SA y las dimensiones de miedos, presentando la ASI-somática una asociación más estrecha con los miedos sangreinyección-daño y agorafóbicos, y la ASI-social con los miedos sociales.

\section{DISCUSIóN}

A través del presente trabajo hemos pretendido estudiar dos aspectos relacionados. Por una parte, deseábamos probar la validez predictiva de la ASI (i.e., predicción del nivel de miedos) partiendo de una muestra amplia de sujetos no clínicos. Esta cuestión sólo habia sido investigada parcialmente en dos trabajos (Lilienfeld, 1997; Reiss et $\left.a_{l}, 1986\right)$, pero con muestras muy pequeñas de sujetos y sin 
considerar la estructura multidimensional de la ASI. En segundo lugar, pretendlamos proporcionar datos adicionales sobre la posible separación entre el rasgo de ansiedad y SA. Para examinar estos objetivos se establecieron tres hipótesis que comentamos a continuación a la luz de los datos obtenidos.

Según nuestra primera hipótesis, la SA deberla relacionarse de forma única con el nivel de miedos y de modo superior al rasgo de ansiedad $y$ otras variables psicológicas relevantes (variables de personalidad, depresión, y afectividad). Tal y como se desprende de los datos que presentamos en la Tabla 2 se puede concluir que la ASI correlaciona con el nivel de miedos de forma más especifica que el rasgo de ansiedad. Las correlaciones entre el rasgo de ansiedad y el nivel de miedos son muy bajas cuando se controla el efecto de la SA, pero no ocurre lo mismo para las correlaciones entre la SA y los miedos cuando se controla el efecto del rasgo de ansiedad. Estos datos, además de indicar que los miedos se relacionan de forma más estrecha con la SA que con el rasgo de ansiedad, proporcionan un apoyo firme a la conceptuación de la SA como un fenómeno diferente del rasgo de ansiedad. Más aun, si tenemos en cuenta que la correlación entre la SA y el rasgo de ansiedad fue de 0,38 (Sandín et al., 2005), lo que significa que estas dos variables sólo comparten el $14 \%$ de la varianza, tendriamos con ello otra prueba sobre la distinción entre ambos constructos. Estos resultados son consistentes con los referidos recientemente por Sandín et al. (2002b) y Valiente et al. (2002a, 2002b) a partir de muestras de niños y adolescentes en las que se aplicó la versión española de la CASI (Childhood Anxiety Sensitivity Index). Las correlaciones entre la SA y los miedos (tras controlar el efecto del rasgo de ansiedad) varian en función de las dimensiones de ambos constructos, lo cual garantiza la conveniencia de emplear las subescalas de la ASI (véase Sandin et al., 2004, 2005), aparte de la puntuación total de SA. Así por ejemplo, vemos que la ASI-somática es la subescala que parece tener una mayor contribución en el nivel general de miedos, en los miedos de sangre-inyeccióndaño y en los temores agorafóbicos, mientras que la subescala ASIsocial se relaciona de forrna más estrecha con los miedos sociales.

En nuestra segunda hipótesis se indicaba que la ASI debería predecir el nivel de miedos mejor que el rasgo de ansiedad $u$ otras variables 
emocionales o de personalidad. Hemos constatado, a este respecto, que únicamente la ASI es capaz de predecir el nivel general de miedos en un análisis de regresión múltiple que incluye también otros predictores como el rasgo de ansiedad, el afecto negativo, el neuroticismo y la depresión (aunque también es significativo el efecto de la extraversión y el neuroticismo, su contribución es minima) (véase la Tabla 3). Estos datos apoyan la evidencia obtenida previamente con poblacion infantil por nuestro grupo sobre la capacidad que posee la SA para predecir los miedos (Sandín et al., 2002b). Los datos también son congruentes con la evidencia de validez incremental de la ASI obtenida con muestras de participantes adultos no clínicos (Lilienfeld, 1997; Reiss et al., 1996). Aparte de proporcionar apoyo a la validez predictiva de la ASI, estos datos demuestran una vez más la distinción entre el rasgo de ansiedad y la SA, y proporcionan evidencia adicional a los hallazgos sobre esta cuestión basados en pacientes con trastomos de ansiedad y análisis de validez discriminante (Taylor et al., 1991).

Finalmente, la tercera hipótesis se basaba en la predicción diferencial de los miedos a partir de las subescalas de la ASI. Según esta tercera hipótesis las dimensiones de la ASI deberian predecir diferenclalmente los tipos de miedos. En concreto, hipotetizamos que la ASI-somática deberla predecir más especificamente los miedos de los tipos agorafobia y sangre-inyección-dano, mientras que la ASIsocial deberla predecir mejor los miedos de tipo social. Basándonos en los datos obtenidos en relación con esta hipótesis (véase la Tabla 4), podriamos afirmar que se cumple esta tercera hipotesis ya que: (1) los miedos del tipo sangre-inyección-daño son predichos únicamente por la subescala ASI-somática, (2) los miedos sociales son mejor predichos por la ASI-social (las dos restantes subescalas contribuyen muy poco en la predicción de estos miedos), y (3) los miedos agorafobicos son mejor predichos por la ASI-somática (las subescalas cognitiva y social aportan escaso poder predictivo). Esto indica que podrla ser conveniente en el futuro tener en cuenta estas dimensiones a la hora de valorar las relaciones entre la SA y los miedos y los trastornos de ansiedad (tanto en lo que concieme a la predicción como a la evolución de los miedos y otros trastornos asociados a la ansiedad). El hecho de que exista una estructura jerárquica de la ASI, y en concreto un factor general de orden superior, el cual explica una 
proporción importante de la varianza en las puntuaciones de la ASI, sugiere también que el empleo de la puntuación global de la escala es apropiado para obtener una indicación del constructo unitario de SA (representado por el factor general) (Sandin et al., 2004). Estos datos, por otra parte, apoyan la evidencia obtenida con población clínica por Zinbarg et al. (1997) y Brown et al. (2003), ya que hallaron una relación más especifica del trastorno de pánico y la fobia social con las subescalas de la ASI somática y social, respectivamente.

A modo de conclusión, en el presente estudio se aporta nueva evidencia sobre la distinción entre la SA y el rasgo de ansiedad, cuestión que ha venido suscitando algunas polémicas (véase, p.ej., Lilienfeld, 1996). Los datos que aqui presentamos complementan, a este respecto, las aportaciones basadas en análisis factorial (Sandin et al., 2001; Taylor et al., 1991) y validez discriminante de la ASI respecto a los trastornos de ansiedad (Taylor, 1999). Por otra parte, según se desprende de algunos estudios (Sandín et al., 2005; Taylor, 1999), la varianza compartida entre ambos constructos es muy baja, lo cual pone en duda la hipótesis, a veces sugerida, de que la SA podria ser un componente del rasgo de ansiedad. Otro aspecto importante que deriva de nuestro trabajo es la validez de la consideración multidimensional de la ASI, un fenómeno ya puesto de relieve por nuestro grupo (Sandín et al., 2004, 2005), que necesaniamente deberá ser contrastada en futuros estudios. Finalmente, el presente trabajo, además de aportar nueva información sobre la capacidad predictiva de la SA respecto a los niveles de miedo, especialmente por la asunción de distintos tipos de miedos (agorafóbicos, sociales y sangre-inyección-daño) y de SA (somática, cognitiva y social), proporciona nueva evidencia a favor de la validez de la versión española de laASI, la cual sin duda podrá ser de gran utilidad clírica por su posible relación diferencial con los distintos constructos y manifestaciones relacionados con la ansiedad (p.ej., Belloch, Morillo y García-Soriano, 2006; Gimeno y Ezquerro, 2006; Nuevo, 2005; Sandin, 2005; Sandín, Rodero, Santed y García-Campayo, 2006).

\section{REFERENCIAS BIBLIOGRÁFICAS}

Belloch, A., Morillo, C., y Garcia-Soriano, G. (2006). Obsession subtypes: Relationships with obsessive-compulsive symptoms, 
dysfunctional beliefs and thought control strategies. Revista de Psicopatología y Psicología Clinica, 11, 65-78.

Brown, M., Smits, J.A.J., Powers, M.B., y Telch, M.J. (2003). Differential sensitivity of the three ASI factors in predicting panic disorder patients' subjective and behavioural response to hyperventilation challenge. Joumal of Anxiety Disorders, 17, 583-591.

Chorot, P., y Sandin, B. (1991). Escala de depresión CESDS. Madrid: UNED (policopiado).

Chorot, P., Sandin, B., Valiente, R.M., Santed, M.A., y Romero, M. (1997). Actitud hacia la enfermedad, ansiedad y sintomatología somática en pacientes con trastorno de pánico e hipocondria. Revista de Psicopatologia y Psicologia Clínica, 2, 123-136.

Ensel, W.M. (1986). Measuring depression: The CES-D Scale. En N. Lin, A. Dean y W. Ensel (Eds.), Social support, life events, and depresion (pp. 51-70). New York: Academic Press.

Francis, L.J., Brown, L.B., y Philipchalk, R. (1992). The development of an abbreviated form of the Revised Eysenck Personality Questionnaire (EPQR-A): Its use among students in England, Canada, the U.S.A and Australia. Personality and Individual Differences, 13, 443-449.

Gimeno, F., y Ezquerro, M. (2006). Intervención psicológica en un caso de evitación interoceptiva en el deporte. Revista de Psicopatología y Psicologia Clínica, 11, 99-106.

Lilienfeld, S.O. (1996). Anxiety sensitivity is not distinct from trait anxiety. In R.M. Rapee (Ed.), Current controversies in the anxiety disorders (pp. 228-244). New York: Guilford Press.

Lillenfeld, S.O. (1997). The relation of anxiety sensitivity to higher and lower order personality dimensions: Implications for the etiology of panic attacks. Joumal of Abnormal Psychology, 106, 539-544.

McNally, R.J. (1996). Desarrollos recientes en el tratamiento del trastomo de pánico. Revista de Psicopatologia y Psicología Clinica, 1, 91-103. Marks, I., y Mathews, A.M. (1979). Brief standard self-rating for phobic patients. Behaviour Research and Therapy, 17, 263-267.

Nuevo, R. (2006). Sensibilidad y especificidad del Cuestionario de Preocupación y Ansiedad para la detección del trastomo de ansiedad generalizada. Revista de Psicopatologia y Psicologia Clinica, 11, 135-142. 
Pelechano, V. (1981). Miedos infantiles y terapia familiar-natural. Valencia: Alfaplús.

Pelechano, V. (1984). Programas de intervención psicológica en la infancia. Valencia: Alfaplús.

Reiss, S. (1991). Expectancy model of fear, anxiety, and panic. Clinical Psychology Review, 11, 141-153.

Reiss, S., Peterson, R.A., Gursky, D.M. y McNally, R.J. (1986). Anxiety sensitivity, anxiety frequency, and the prediction of fearfulness. Behaviour Research and Therapy, 24, 1-8.

Sandin, B. (1997). Ansiedad, miedos y fobjas y nihos y adolescentes. Madrid: Dykinson.

Sandín, B. (1999). El Estrés psicosoclal: Conceptos y consecuencias clfnicas. Madrid: Klirik.

Sandín, B. (2005). Evitación interoceptiva: Nuevo constructo en el campo de los trastomos de ansiedad. Revista de Psicopatologla y Psicologla Clinica, 10, 103-114.

Sandin, B., Chorot, P., y McNally, R.J. (2001). Anxiety sensitivity index: Normative data and its differentiation from trait anxiety. Behaviour Research and Therapy, 39, 213-219.

Sandin, B., Chorot, P., Lostao, L., Joiner, T.E., Santed, M.A., y Valiente, R.M. (1999). Escalas PANAS de afecto positivo y negativo: Validación factorial y convergencia transcultural. Psicothema, 11, 37-51.

Sandin, B., Chorot, P., Santed, M.A., y Valiente, R.M. (2002a). Análisis factorial confirmatorio del Indice de Sensibilidad a la Ansiedad para Niños. Psicothema, 14, 333-339.

Sandín, B., Chorot, P., Valiente, R.M., y Santed, M.A. (1998). Frecuencia e intensidad de los miedos en los niños: Datos normativos. Revista de Psicopatologia y Psicologla Clinica, 3, 15-25.

Sandín, B., Chorot, P., Valiente, R.M., y Santed, M.A. (2002b). Relación entre la sensibilidad a la ansiedad y el nivel de miedos en niños. Psicologla Conductual, 10, 107-120.

Sandin, B., Chorot, P., Valiente, R.M., Santed, M.A., y Lostao, L. (2004).

Dimensiones de la sensibilidad a la ansiedad: evidencia confirmatoria de la estructura jerárquica. Revista de Psicopatologfa y Psicologla Clinica, 9, 19-33.

Sandín, B., Chorot, P., Valiente, R.M., Santed, M.A., y Sánchez-Arribas, C. (1999a). Estructura factoriai de la Escala de Ansiedad Social 
para Niños-Revisada (SASC-R). Revista de Psicopatologla y Psicologla Clínica, 4, 103-113.

Sandin, B., Rodero, B., Santed, M.A., y Garcla-Campayo, J. (2006). Sucesos vitales estresantes y trastomo de pánico: Relación con la ocurrencia del trastomo de pánico, la gravedad clínica y la agorafobia. Revista de Psicopatologia y Psicologia Clínica, 11, 179-190.

Sandin, B., Valiente, R.M., y Chorot, P. (1999). Material de apoyo para la evaluación de los miedos y fobias. En B. Sandín (Ed.), Las fobias espec/ficas (pp. 161-188). Madrid: Klinik.

Sandin, B., Valiente, R.M., Chorot, P., y Santed, M.A. (2005).

Propiedades psicométricas del Indice de Sensibilidad a laAnsiedad. Psicothema, 17, 478-483.

Sandin, B., Valiente, R.M., Chorot, P., Olmedo, M., y Santed, M.A. (2002c). Versión española del cuestionario EPQR-Abreviado (EPQR-A) (I): Análisis exploratorio de la estructura factorial. Revista de Psicopatología y Psicología Clínica, 7, 195-205.

Sandin, B., Valiente, R.M., Chorot, P., Santed, M.A., y Sánchez-Arribas,

C. (1999b). Escala de Ansiedad Social para Niños-Revisada (SASC-R): Fiabilidad, validez y datos normativos. Análisis y Modificación de Conducta, 25, 827-847.

Sandin, B., Valiente, R.M., Olmedo, M., Chorot, P., y Santed, M.A. (2002d). Versión espańola del cuestionario EPQR-Abreviado (EPQR-A) (II): Replicación factorial, fiabilidad y validez. Revista de Psicopatologia y Psicologia Clínica, 7, 207-216.

Spielberger, C.D., Gorsuch, R.R., y Lushene, R.E. (1982). STAl. Cuestionario de Ansiedad Estado/Rasgo. Madrid: Técnicos Especialistas Asociados (TEA).

Stevens, J. (1996). Applled multivariate statistics for the social sciences (3rd ed.). Mahwah, NJ: LEA.

Stewart, S.H., Taylor, S., y Baker, J.M. (1997). Gender differences in dimensions of anxiety sensitivity. Joumal of Anxiety Disorders, 11. 179-200.

Taylor, S. (Ed.) (1999). Anxiety sensitivity: Theory, research, and treatment of the fear of anxiety. Mahwah, NJ: LEA.

Taylor, S., y Cox, B.J. (1998). An expanded Anxiety Sensitivity Index: Evidence for a Hierarchic structure in a clinical sample. Journal of Anxiety Disorders, 12, 463-483. 
Taylor, S., Koch, W.J., y Crockett, D.J. (1991). Anxiety sensitivity, trait anxiety, and the anxiety disorders. Joumal of Anxiety Disorders, 5 , 293-311.

Taylor, S., Koch, W.J., Woody, S., y McLean, P. (1996). Anxiety sensitivity and depression: How are they related? Journal of Abnormal Psychology, 105, 474-479.

Valiente, R.M., Sandin, B., y Chorot, P. (2002a). Miedos comunes en niños y adolescentes: Relación con la sensibilidad a la ansiedad, el rasgo de ansiedad, la afectividad negativa y la depresión. Revista de Psicopatologla y Psicologla Clínica, 7, 61-70.

Valiente, R.M., Sandin, B., y Chorot, P. (2002b). Miedos comunes en niños y adolescentes: Su relación con la sensibilidad a la ansiedad y otras emociones negativas. Psiquis, 23, 217-225.

Valiente, R.M., Sandín, B., Chorot, P., y Tabar, A. (2002). Diferencias sexuales en la prevalencia e intensidad de los miedos durante la infancia y la adolescencia: datos basados en el FSSC-R. Revista de Psicopatologla y Psicologla Clínica, 7, 103-113.

Valiente, R.M., Sandín, B., Chorot, P., y Tabar, A. (2003). Diferencias según la edad en la prevalencia e intensidad de los miedos durante la infancia y la adolescencia: datos basados en el FSSC-R. Psicothema, 15, 414-419.

Watson, D., Clark, L.A., y Tellegen, A. (1988). Development and validation of brief measures of positive and negative affect: The PANAS scales. Joumal of Personality and Social Psychology, 54, 1063-1070.

Zinbarg, R.E., Barlow, D.H., y Brown, T.A. (1997). Hierarchical structure and general factor saturation of the Anxiety Sensitivity Index: Evidence and implications. Psychological Assessment, 9, 277-284.

Zvolensky, M.J., Arrindell, W.A., Taylor, S., Bouvard, M., Cox, B.J., Stewart, S.H., Sandin, B., Jurado Cardenas, S., y Eifert, GH. (2003). Anxiety sensitivity in six countries. Behaviour Research and Therapy, 41, 841-859. 